\title{
Hybrid Films Based on a Bridged Silsesquioxane Doped with Goethite and Montmorillonite Nanoparticles as Sorbents of Wastewater Contaminants
}

\author{
Carolina V. Waiman, ${ }^{1}$ Ignacio E. dell'Erba, ${ }^{2}$ Carlos A. Chesta, ${ }^{1}$ and María Lorena Gómez ${ }^{1}$ \\ ${ }^{1}$ Departamento de Química, Universidad Nacional de Río Cuarto y CONICET, Campus Universitario, 5800 Río Cuarto, Argentina \\ ${ }^{2}$ Institute of Materials Science and Technology (INTEMA), University of Mar del Plata and National Research Council (CONICET), \\ J. B. Justo 4302, 7600 Mar del Plata, Argentina
}

Correspondence should be addressed to María Lorena Gómez; mlgomez@exa.unrc.edu.ar

Received 2 November 2015; Accepted 24 December 2015

Academic Editor: Giovanni Bongiovanni

Copyright ( 2016 Carolina V. Waiman et al. This is an open access article distributed under the Creative Commons Attribution License, which permits unrestricted use, distribution, and reproduction in any medium, provided the original work is properly cited.

\begin{abstract}
The synthesis and characterization of silsesquioxane (SSO) films with pendant dodecyl groups and doped with goethite (Gt) or montmorillonite (MMT) nanoparticles were carried out and the new materials tested as sorbents of diverse contaminants. The synthetic method used yielded SSO films with the inorganic substrates homogeneously distributed within the polymeric matrices. The new materials were characterized by SEM, FTIR, XRD, and DSC and tested to evaluate their capability for adsorbing metallic cations, organic dyes, and phosphate, frequent contaminants of industrial effluents. All films were found suitable for removing metallic cations. Results also showed that the SSO films undoped and doped with Gt are primarily apt for anionic compounds removal. Although the SSO films doped with MMT are capable of removing cationic contaminants from aqueous samples, the stiffness of the SSO matrix hinders MMT properties as an adsorbent. The possibility of dispersing nanoparticulate systems in the stable and chemically inert SSO matrices simplifies their application for contaminant removal, particularly because it makes the separation process of the absorbed pollutant from the treated medium easier.
\end{abstract}

\section{Introduction}

Bridged silsesquioxanes (SSO) are a family of organic-inorganic hybrid materials obtained from hydrolysis and polycondensation of monomers containing organic groups bridging two (or more) trialkoxysilanes or trichlorosilanes [1-3]. Depending on the type of precursor used for the synthesis, SSO may have different properties such as high chemical and thermal resistance, biocompatibility, and luminescence. Many of these SSO also allow the homogeneous dispersion of other substances (dyes, coordination complexes, porphyrins, etc.) and particulate material (gold nanoparticles, clays, etc.). Interestingly, craft hybrid materials based on organically modified clays have been developed and manufactured along human history for multiple industrial, commercial, environmental, artistic, and social uses [4]. SSO doped with different substrates have found interesting technological applications $[5,6]$. Among these, SSO can be successfully used to synthesize sorbents materials [7]. Several bridging groups containing carbamate, ammonium, thiourea, malonamide, sulfone, or sulfoxide groups, among other, were employed as active sorbents for heavy metals and lanthanides [810]. Furthermore, SSO functionalized with amine and thiol groups demonstrate being effective for the adsorption of volatile organic compounds from the air [11].

In recent years, we were able to incorporate diverse substrates into SSO films achieving stable dispersions and materials with different properties. For instance, it was possible to synthesize SSO films doped with safranin, in which the light energy collected by the matrix is efficiently transferred to the fluorescent dye [12]. It was also possible to synthesize new SSO films with pendant dodecyl chains able to disperse metallic nanoparticles [13, 14], gold thiolates [15], and silver thiolates [16]. On the other hand, the incorporation of porphyrin or $\operatorname{Re}(\mathrm{I})$ complexes in these materials proved to be very effective in generating reactive oxygen species 
<smiles>O=C([O-])c1ccccc1-c1c2cc(Br)c(=O)c(Br)c-2oc2c(Br)c(O)c(Br)cc12</smiles>

Eosin y<smiles>CN(C)c1ccc2nc3ccc(=[N+](C)C)cc-3sc2c1</smiles>

Methylene blue<smiles>O=C(OC1(c2cc(I)c(Cl)c(Cl)c2Cl)c2cc(I)c(O)cc2OC2C=C(O)C(I)=CC21)c1cc(I)c(O)c(Cl)c1Cl</smiles>

Rose bengal<smiles>O=P([O-])(O)O</smiles>

Phosphate<smiles>O=C([O-])c1ccccc1-c1c2ccc(=O)cc-2oc2cc(O)ccc12</smiles>

Fluorescein

Scheme 1: Molecular structure of dyes and water contaminants tested in this study.

allowing the photodynamic inactivation of fungi such as $C$. albicans [17] or the photooxidation of organic compound [18].

It is well known that goethite (Gt), an iron oxide, shows a high capacity to adsorb contaminants from aqueous samples [19]. Montmorillonite (MMT) is natural nanosized clay that has also been used to remove water pollutants [20]. Hence, we report herein the synthesis and characterization of SSO films doped with Gt or MMT and prove their capacity for removing diverse types of water contaminants [21-23].

The structure of the organic dyes and inorganic contaminants probed in this study is shown in Scheme 1. Heavy metals are usually discharged from industries, mining operations, fertilizer industry, batteries, and so forth; phosphate anions are mostly introduced by agriculture practices as fertilizer, and synthetic dyes are discharged into the environment mainly by human activities such printing inks, paints, plastic, food, cosmetic, and textile industries [24].

\section{Experimental}

2.1. Materials. Dodecylamine (DA) (98\%, Fluka) and glycidoxypropyltrimethoxysilane (GPTMS) (97\%, Sigma) were used without further purification.

Tetrahydrofuran (THF) (Sintorgan) was refluxed with $\mathrm{KOH}$ and distilled over $4 \AA$ molecular sieves. Water was purified by using a Millipore Milli-Q system. Formic acid (Sintorgan) was used as received.

Adsorbates studied were phosphate $\left(\mathrm{Na}_{2} \mathrm{HPO}_{4}\right)(99 \%$ purity, Sigma-Aldrich), copper $\left(\mathrm{Cu}\left(\mathrm{NO}_{3}\right)_{2} \cdot 3 \mathrm{H}_{2} \mathrm{O}\right)(99 \%$, Anedra), cadmium $\left(\mathrm{Cd}\left(\mathrm{NO}_{3}\right)_{2} \cdot 4 \mathrm{H}_{2} \mathrm{O}\right)$ (99\% purity, Riedel-de Haën), eosin y (99\%, Sigma-Aldrich), rose bengal (95\%, Sigma-Aldrich), fluorescein (95\%, Sigma-Aldrich), and methylene blue (95\%, Cicarelli).

2.2. Characterization Techniques. XRD patterns of films were obtained in the $2 \theta$ range comprised between $2^{\circ}$ and $60^{\circ}$, using
Rigaku D-Max III-C diffractometer applying monochromated Co $\mathrm{K} \alpha$ radiation at $35 \mathrm{kV}$ and $15 \mathrm{~mA}$.

ATR-FTIR spectra were obtained using a Nicolet Nexus 470 FTIR spectrometer equipped with a DTGS detector, a SMART-ARK ATR accessory, and a ZnSe crystal (area: $10 \times$ $72 \mathrm{~mm}$, incident angle: $45^{\circ}$, total reflections: 12 ).

Differential scanning calorimetry (DSC) thermograms were obtained with a DSC-50 Shimadzu equipment at a heating rate of $10^{\circ} \mathrm{C} / \mathrm{min}$, under $\mathrm{N}_{2}$ flow.

To visualize the morphology and structure of the aggregated nanoparticles, a Scanning Electron Microscope JEOL JSM-6460LV was employed. The samples were loaded on the surface of an aluminum SEM specimen holder and sputter coated with Au-Pd for $35 \mathrm{~s}$ before observation. A working distance about $20-25 \mathrm{~mm}$, an accelerating voltage of $15 \mathrm{kV}$, and a chamber pressure of $10^{-9}$ Torr were found to be suitable for obtaining high-resolution images of hydrogel samples. The magnification in this study was $2000 \mathrm{x}$.

Elements in the films were analyzed by X-Ray Energy Dispersion Spectroscopy (EDS). The system used was EDAX Genesis XM4-Sys 60, equipped with Multichannel Analyzer (MCA) EDAX mod EDAM IV, Sapphire Si(Li) detector and Super Ultra Thin Window of Be, and EDAX Genesis V 5.11 software.

2.3. Goethite (Gt) Synthesis and Characterization. Gt particles were synthesized following a method similar to that described by Atkinson et al. [25]. Briefly, $160 \mathrm{~mL}$ of $5 \mathrm{M} \mathrm{NaOH}$ solution was added dropwise to $800 \mathrm{~mL}$ of $0.1 \mathrm{M} \mathrm{Fe}\left(\mathrm{NO}_{3}\right)_{3}$ with continuous stirring at room temperature to get a mixture of iron oxides. Carbon dioxide contamination was minimized during the synthesis by bubbling water-saturated $\mathrm{N}_{2}$. The resulting ferrihydrite suspension was aged at $60^{\circ} \mathrm{C}$ for 3 days and then washed with Milli-Q water to remove dissolved sodium nitrate and excess sodium hydroxide to finally obtain a suspension of pure Gt. 


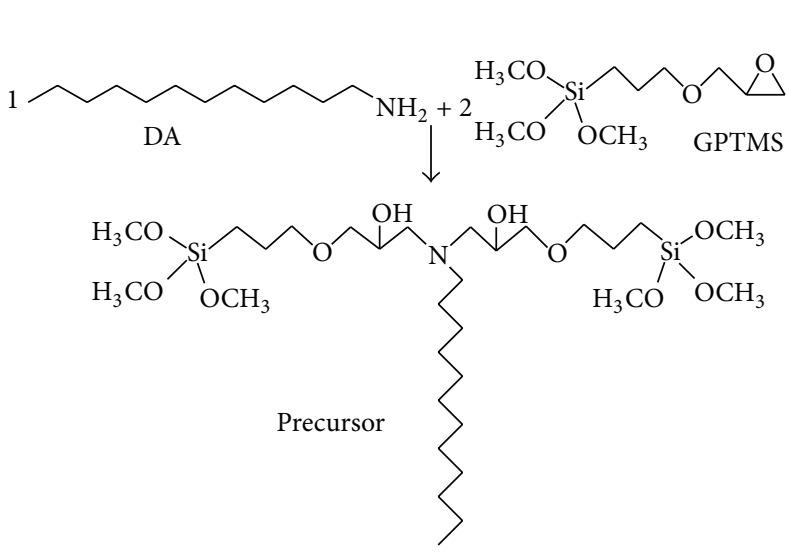

(a)

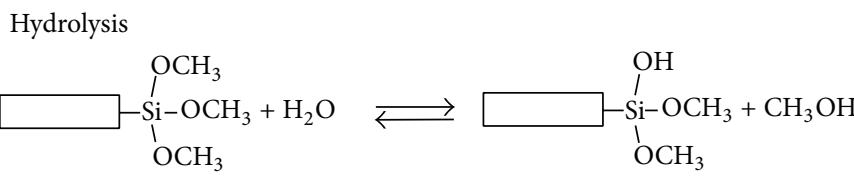

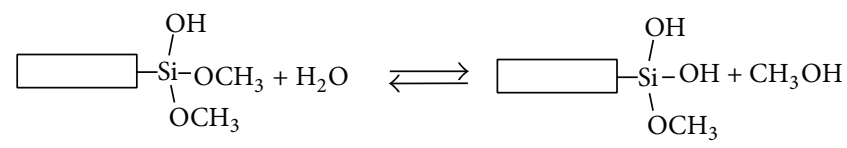

Condensation

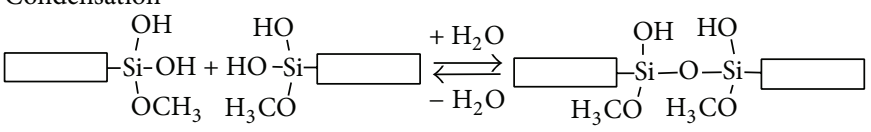

(b)

Scheme 2: Schematic representation of the synthesis of SSO films. Synthesis of SSO precursor (a). Hydrolysis and polycondensation of silane groups (b).

The so-prepared Gt was stored as a stock suspension at $\mathrm{pH}$ 5. An aliquot of this suspension was dried for characterization. X-ray powder diffraction (XRD), infrared (IR) spectroscopy, and electron microscopy were used to confirm Gt structure. Gt particles are acicular and according to Kosmulski et al. their particle size is around $94 \mathrm{~nm} \times 10 \mathrm{~nm}$ [26]. The $\mathrm{N}_{2}$-BET surface area was $40 \mathrm{~m}^{2} / \mathrm{g}$ measured with a Quantachrome Nova 1200e instrument. Gt is a variablecharge iron oxide with an isoelectric point (IEP) around $\mathrm{pH}$ 8.3-9.0. As a consequence of protonation-deprotonation processes on surface groups, at $\mathrm{pH}<\mathrm{IEP}$ Gt particles have positive charge and at $\mathrm{pH}>$ IEP they have negative charge [21].

2.4. Montmorillonite (MMT). Natural occurring MMT nanoparticles were obtained from Northern Patagonia (Argentina). The general characterization of this material was already reported by Ilari et al. [27]. The material is composed mainly of Na-MMT ( $99.4 \%$ purity), having a cation exchange capacity (CEC) of $0.91 \mathrm{mEq} / \mathrm{g}$, a specific surface area of $738 \mathrm{~m}^{2} / \mathrm{g}$, and a BET surface area of $2 \mathrm{~m}^{2} / \mathrm{g}$. This clay forms aggregates with an average size $\sim 200 \mathrm{~nm}$; and they contain a structural negative charge independent of $\mathrm{pH}$ [28].

2.5. Adsorption Experiments. Adsorption experiments were performed by batch experiments and by kinetics measurements. All experiments were conducted at $\mathrm{pH} 6.0$ in $\mathrm{NaCl}$ $0.01 \mathrm{M}$ as supporting electrolyte, conditions that resemble natural aquifers, the final "place" of most industrial wastes.

Batch experiments were carried out in polypropylene tubes by submerging pieces of films of the same mass in the adsorbate solution. Adsorbates tested in this study were dyes (eosin y, rose bengal, fluorescein, and methylene blue), phosphate anions, and divalent metal cations (copper and cadmium). The tubes were shaken during 24 hours; then the films were removed and the adsorbates remaining in the solution not adsorbed were quantified. Dye's absorptions were recorded with UV-Vis absorption spectrophotometer (HP 8452A, Hewlett-Packard) at $517 \mathrm{~nm}, 549 \mathrm{~nm}, 452 \mathrm{~nm}$, and $662 \mathrm{~nm}$, for eosin y, rose bengal, fluorescein, and methylene blue, respectively. The concentration of phosphate anion was quantified by the molybdenum blue method developed by Murphy and Riley [29] and divalent metal cations were quantified by using an atomic absorption spectrometer (GBC Avanta model B-932).

Kinetic studies were performed by submerging pieces of films of the same weight in a solution of dyes. Absorption spectra of dye solutions were automatically collected at constant time intervals using UV-Vis absorption spectrophotometer (HP 8452A, Hewlett-Packard) equipped with a kinetic software module. The absorption in the maximum against time was recorded at $517 \mathrm{~nm}$ for eosin y and $662 \mathrm{~nm}$ for methylene blue. The adsorption processes were followed during 4 and 8 hours for eosin y and methylene blue, respectively.

\section{Results and Discussion}

3.1. Synthesis of Silsesquioxanes Films. The synthesis of the films based on SSO was reported before [13]. The precursor of SSO was synthesized employing stoichiometric amounts of DA and GPTMS $(1: 2)$. The reaction was carried out in $0.4 \mathrm{M}$ DA solution in THF under nitrogen atmosphere at $58^{\circ} \mathrm{C}$, until complete conversion of the reagents was attained $(\sim 48 \mathrm{~h})$. The hydrolysis and condensation process was carried out at room temperature, employing $0.1 \mathrm{M}$ solutions of the SSO precursor in THF, adding the appropriate amount of water and catalyst in order to obtain the molar ratio $\mathrm{Si} / \mathrm{HCOOH} / \mathrm{H}_{2} \mathrm{O}=1 / 0.1 / 3$. Solutions were casted in polyacetal recipients of $5 \mathrm{~cm}$ diameter with an initial height of liquid of $\sim 5 \mathrm{~mm}$ and placed in an oven at $30^{\circ} \mathrm{C}$ for $24 \mathrm{~h}$. Hydrolysis and condensation reactions took place during solvent evaporation. Films obtained were transparent and showed a pale yellow color. The low content of acid used as catalyst diminishes the polycondensation rate and permits the bridged groups to self-organize within the matrix resulting in flexible thin films. Molecular structures and schematic representation of the synthesis are presented in Scheme 2. 


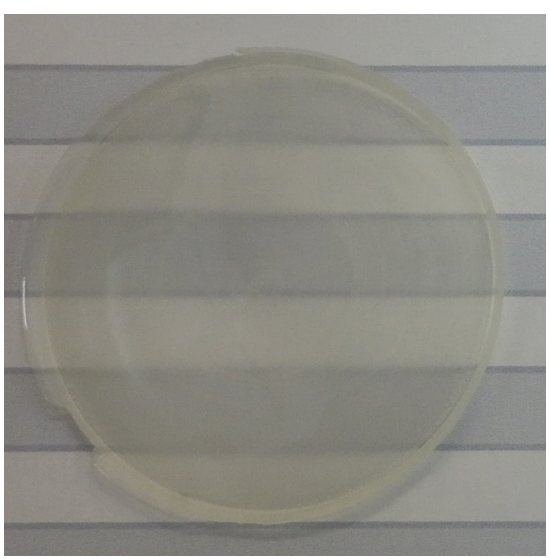

(a) Undoped

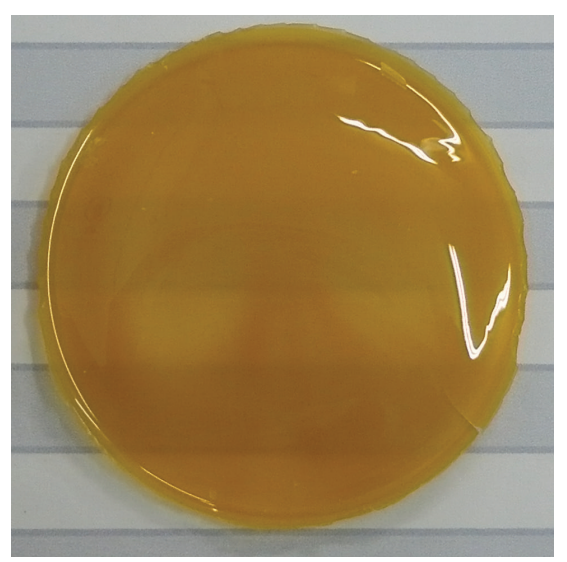

(b) SSO-Gt $(500 \mu \mathrm{L}, 20 \mathrm{~g} / \mathrm{L})$

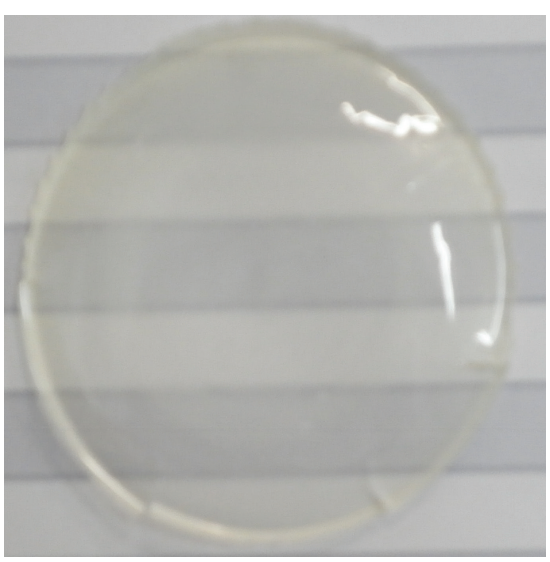

(c) SSO-MMT $(500 \mu \mathrm{L}, 5 \mathrm{~g} / \mathrm{L})$

FIGURE 1: Photographs of flexible transparent thin films of SSO (a); SSO-Gt (b); and SSO-MMT (c).

SSO

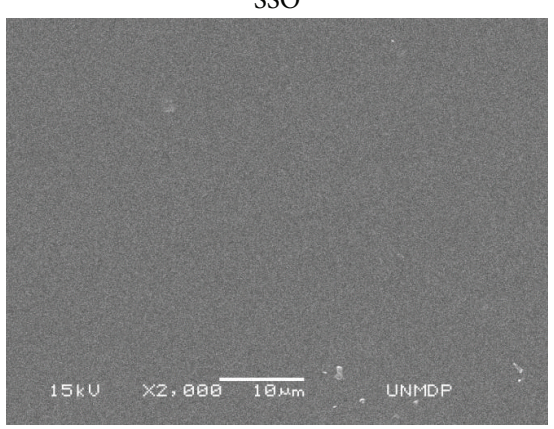

(a)

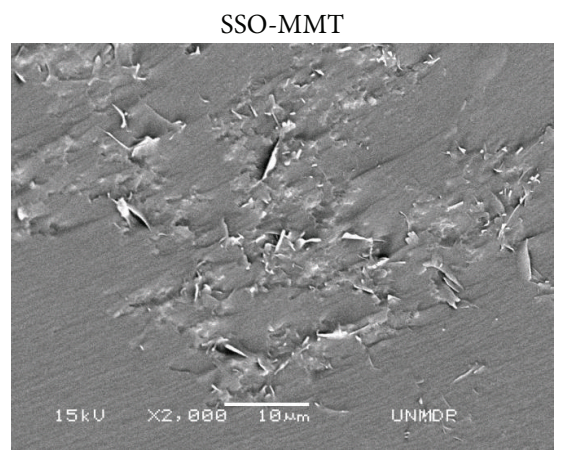

(b)

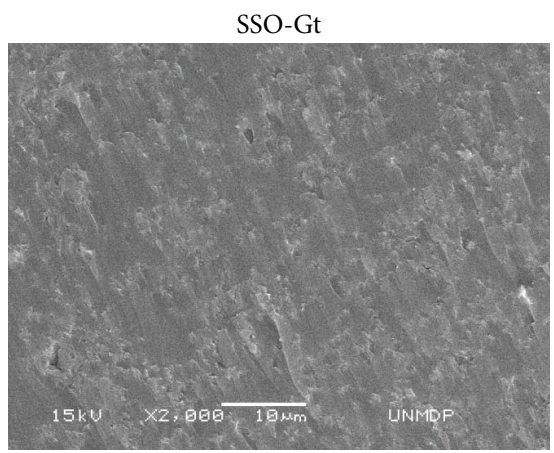

(c)

FIGURE 2: SEM micrographs of undoped SSO film (a), SSO-MMT film (b), and SSO-Gt film (c).

The synthesis of SSO doped films was performed following a similar procedure. In this case, $0.5 \mu \mathrm{L}$ of stock suspension of Gt $(20 \mathrm{~g} / \mathrm{L})$ or of MMT $(5 \mathrm{~g} / \mathrm{L})$ was incorporated with the prepolymeric mixture in order to obtain the nanoparticles dispersions in SSO films. Films doped with Gt (SSO-Gt) have a yellowish-brown color losing some transparency, while those doped with MMT (SSO-MMT) retain the characteristics of undoped films. Both types of particles remain strongly retained within the SSO films. This was concluded since no release of Gt or MMT was detected after immersing the films in water for more than 30 days. Photographs of the synthesized materials are shown in Figure 1.

3.2. Characterization. SEM micrographs of the synthesized undoped and doped SSO films are shown in Figure 2. As shown, Gt and MMT are nearly homogenously dispersed in the polymer matrices, although some regions with higher concentration of dopants are detectable. While the presence of MMT produces exfoliated regions on the surface of the film (Figure 2(b)), SSO-Gt films show a homogenous distribution of small particle aggregates in the material (Figure 2(c)).

EDAX was used to identify the overall composition of the different domains: SSO matrix and dopants (Figure 3).
$\mathrm{Au}$ and Pd are always present in all samples and they arise from the metallized deposition treatment of the samples' previous analyses. In the case of SSO doped with Gt, the presence of $\mathrm{Fe}$ is evident and it is uniformly distributed in the polymer matrix (Figure 3(c)). This constitutes a further evidence of the homogeneous dispersion of Gt in the SSO films. As expected, in the case of samples with MMT the presence of $\mathrm{Ca}, \mathrm{Al}$, and $\mathrm{Mg}$ is observed (Figure 3(b)).

In order to complete the characterization of the materials ATR, XRD, DSC, and TGA analyses were performed for the undoped and doped SSO films, as well as for Gt and MMT powders. Figure 4 shows DSC thermograms of the hybrid films and of pure samples of MMT and Gt. The presence of Gt in the films could be confirmed by the transition around $250^{\circ} \mathrm{C}$ that corresponds to thermal transformation of Gt to hematite [30]. This transition, clearly visible for the pure compound, appears as a small peak in the SSOGt doped sample. In the case of MMT, the most common thermal transition associated with this clay is the loss of water $\left(<150^{\circ} \mathrm{C}\right)$ [31], transition that is almost imperceptible in SSO-MMT doped films, probably because of the small concentration of the dopant in these samples. All SSO films show exothermic peaks between 350 and $500^{\circ} \mathrm{C}$ that can be assigned to the thermal irreversible decomposition of 

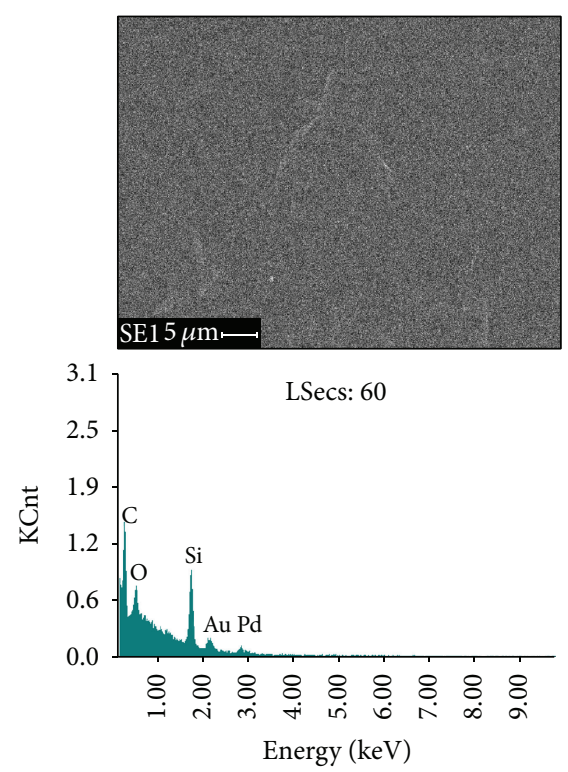

(a) $\mathrm{SSO}$
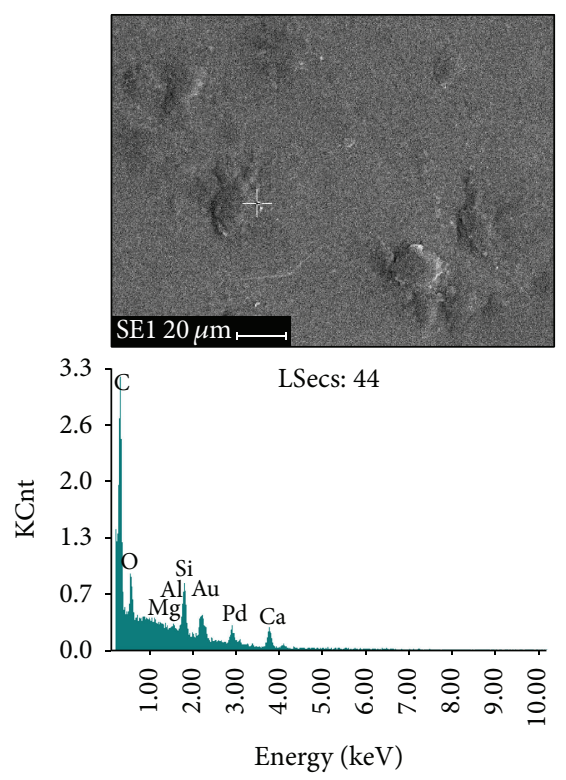

(b) SSO-MMT
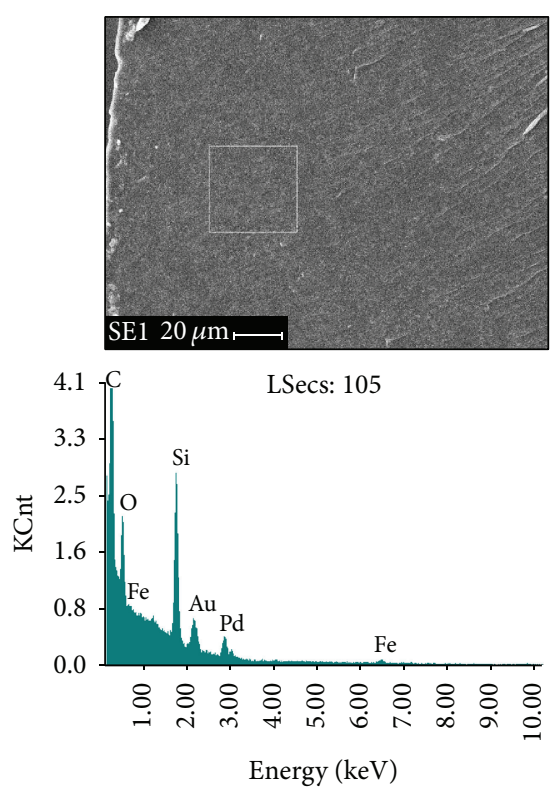

(c) SSO-Gt

FIGURE 3: SEM-EDAX micrographs of undoped SSO film (a), SSO-MMT film (b), and SSO-Gt film (c).

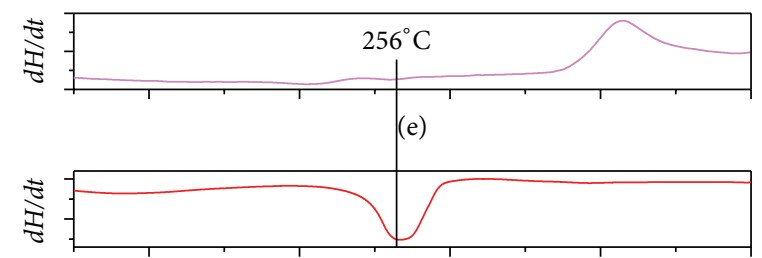

(d)

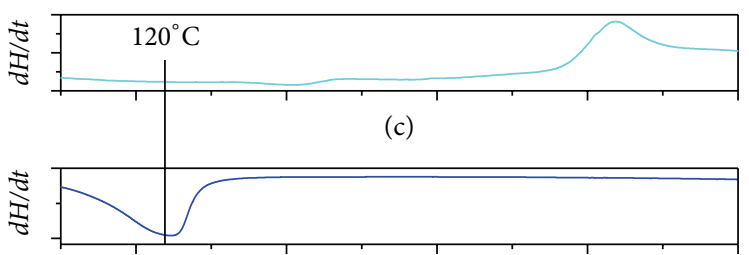

(b)

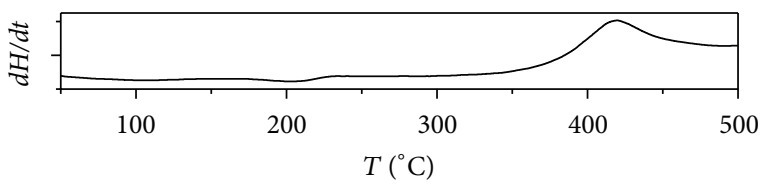

(a)

FIGURE 4: DSC thermogram of the SSO film (a), MMT (b), Gt (d), and the inorganic compound embedded in the polymer: SSO-MMT film (c) and MMT-Gt film (e).

the matrix [32]. These polymeric materials undergo a mass loss of about $70 \%$ after the first scan, but no significant transitions or mass loss was observed in a second scan.

Figure 5 illustrates X-ray diffraction spectra of SSO undoped and doped films and the corresponding Gt and MMT powders. The X-ray diffraction spectrum of the SSO film presents a broad peak near $2 \theta=21.64^{\circ}$, characteristic of amorphous state of this material [16]. In the case of the film doped with Gt (Figure 5(a)), the characteristics peaks of Gt at $2 \theta=21.20^{\circ}, 33.25^{\circ}, 36.60^{\circ}$, and $53.99^{\circ}$ that correspond to $d$ spacings $d_{101}=4.18 \AA, d_{301}=2.69 \AA, d_{111}=2.45 \AA$, and $d_{212}=$ $1.72 \AA$, respectively, are also present in the doped film [33, 34]. In the case of the film doped with MMT (Figure 5(b)), only the characteristic peak at $2 \theta=6.95^{\circ}\left(d_{001}=12.7 \AA\right)$ is observable in the doped SSO film, probably because of the low content of dopant in the material $[31,35]$.

ATR spectra of SSO films doped with Gt and MMT are shown in Figures 6(a) and 6(b), respectively. Spectra of undoped SSO films present two peaks at $1024 \mathrm{~cm}^{-1}$ and $1097 \mathrm{~cm}^{-1}$, characteristics of antisymmetric Si-O-Si vibrations. The presence of a relatively intense peak at $916 \mathrm{~cm}^{-1}$ (assigned to $\mathrm{SiOH}$ groups) indicates a relatively low conversion of the precursor in the polycondensation reaction. The broad band with a maximum at $3370 \mathrm{~cm}^{-1}$ is characteristic of $\mathrm{H}$-bonded $\mathrm{SiOH}$ and $\mathrm{COH}$ groups. The two peaks at $2850 \mathrm{~cm}^{-1}$ and $2920 \mathrm{~cm}^{-1}$ are characteristic stretching vibrations of $\mathrm{CH}_{2}$ groups in extended chains (all-trans conformation) [36].

SSO-Gt (Figure 6(a), blue line) shows the presence of the characteristics vibrations at 796 and $889 \mathrm{~cm}^{-1}$ assigned to $\mathrm{FeO}-\mathrm{H}$ bending of Gt [33]. In SSO-MMT sample (Figure 6(b), green line) the presence of two bands at 1045 and $1635 \mathrm{~cm}^{-1}$ that correspond to $\mathrm{Si}-\mathrm{O}$ stretching and $\mathrm{OH}$-bending of MMT is also observed in the ATR spectrum of the doped film.

3.3. Adsorption Assays. With the aim of assessing the properties of the synthesized materials as sorbents, adsorption experiments for organic dyes, divalent metal cations, and 


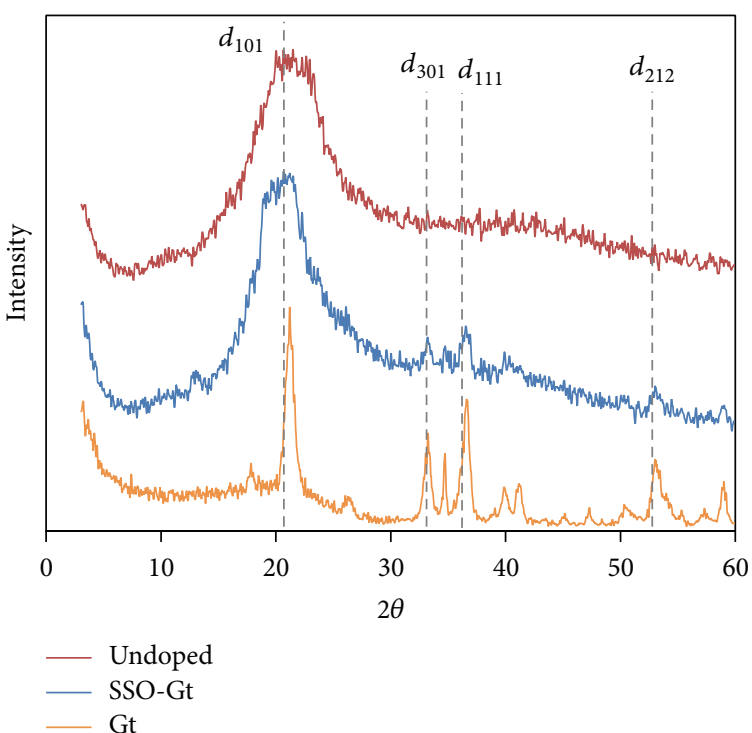

(a)

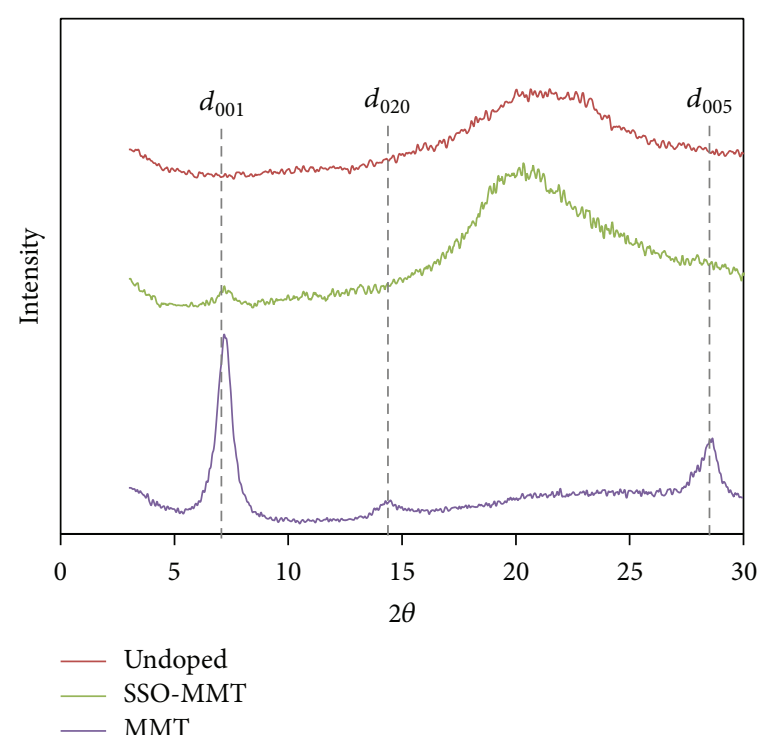

(b)

FIGURE 5: X-ray diffraction spectra of SSO films, Gt, and MMT.

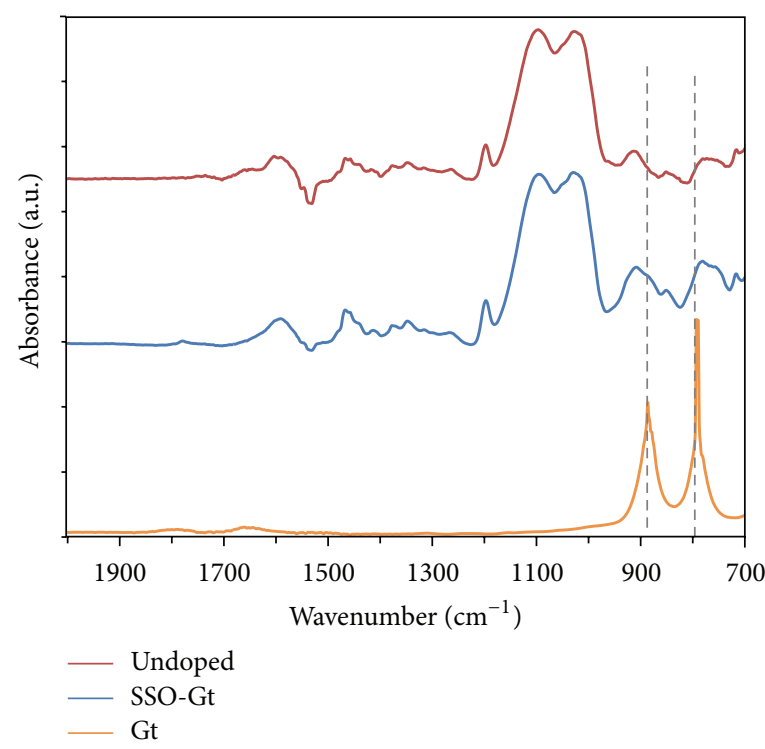

(a)

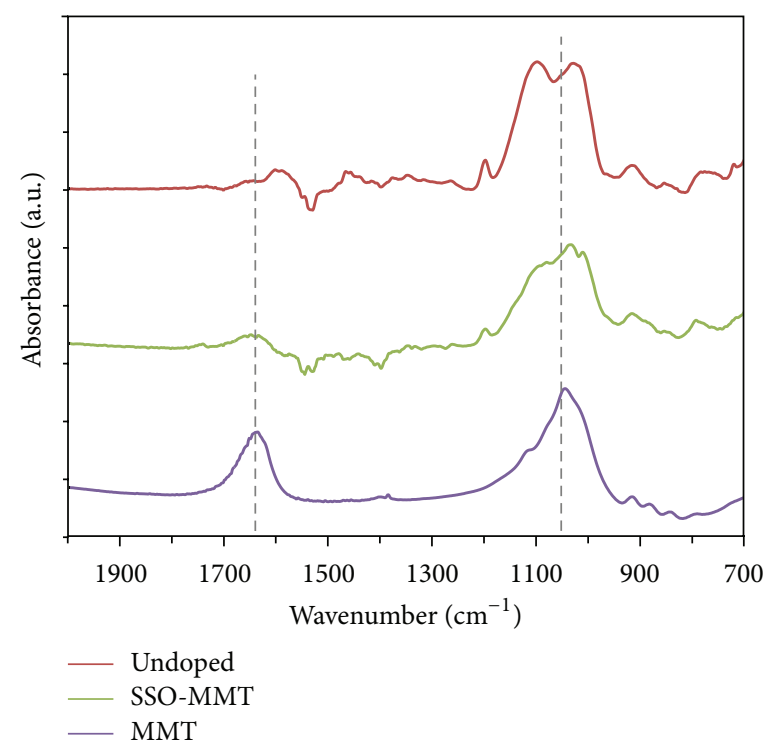

(b)

FIGURE 6: FTIR spectra of SSO films and Gt and MMT dusts.

phosphate were conducted in simulated environment aquifer $(\mathrm{pH}=6.0-0.01 \mathrm{M} \mathrm{NaCl})$.

Figure 7 illustrates the results for eosin y and fluorescein. Test tubes labeled as (0) correspond to the blanks experiments. Tubes labeled as (1-3) show the effect of adding the same mass of SSO (1), SSO-Gt (2), and SSO-MMT (3) films to the solution of dyes after $8 \mathrm{~h}$ in the dark.

As shown in Figure 7, the films become colored due to the retention of dyes and the supernatant becomes nearly colorless compared to control tube (0). Quantitative studies were performed by UV-Vis spectroscopy for dyes, atomic absorption spectroscopy for metal cations, and the molybdenum blue method to quantify phosphate anion [29].

Table 1 collects the adsorbed percentages of the different substrates in SSO, SSO-Gt, and SSO-MMT films.

In the case of metal cations retention around $30 \%$ could be reached with these materials, principally for SSO-MMT films; this might be ascribed to the negatively charged clay surface at the working $\mathrm{pH}[27,28]$.

In the case of dyes, all films seem to be good sorbents; however SSO-Gt retains larger amounts of the anionic compounds, reaching values around $90 \%$ for fluorescein. 


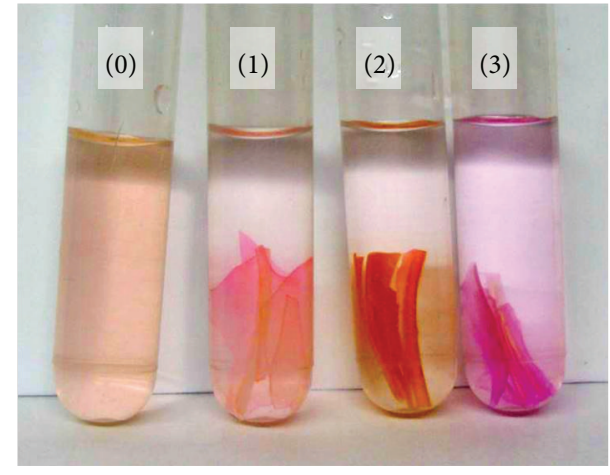

(0) Without adsorbent

(1) SSO
(2) $\mathrm{SSO}-\mathrm{Gt}$

(3) SSO-MMT

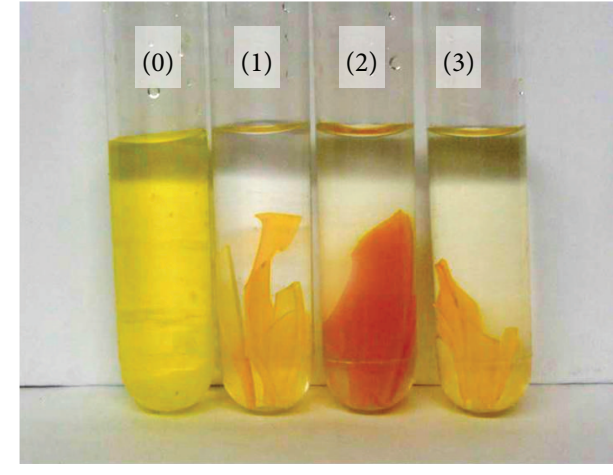

(0) Without adsorbent

(1) SSO

(2) SSO-Gt

(a) Eosin y

(b) Fluorescein

Figure 7: Photograph of eosin y (a) and fluorescein (b) adsorbed on SSO films.

TABLE 1: Determination of contaminants adsorption expressed as percentage of the initial concentration.

\begin{tabular}{|c|c|c|c|c|c|c|c|}
\hline Sorbent & $\mathrm{Cu}^{2+}$ & $\mathrm{Cd}^{2+}$ & Eosin $y$ & Rose bengal & Fluorescein & Methylene blue & Phosphate \\
\hline SSO & 20 & 13 & 40 & 60 & 77 & 3 & 30 \\
\hline SSO-Gt & 30 & 26 & 80 & 81 & 89 & 5 & 60 \\
\hline SSO-MMT & 34 & 32 & 40 & 60 & 80 & 16 & 20 \\
\hline
\end{tabular}

Initial concentration of contaminants: $\mathrm{Cu}^{2+}: 3.0 \mathrm{mg} / \mathrm{L} ; \mathrm{Cd}^{2+}: 0.8 \mathrm{mg} / \mathrm{L} ;$ eosin y: $2.8 \mathrm{mg} / \mathrm{L}$; rose bengal: $3.6 \mathrm{mg} / \mathrm{L}$; fluorescein: $9.0 \mathrm{mg} / \mathrm{L} ; \mathrm{methylene} \mathrm{blue:} 2.6 \mathrm{mg} / \mathrm{L}$; phosphate: $2.3 \mathrm{mg} / \mathrm{L}$.

Sorbent mass: $0.30 \mathrm{~g}$ in $5 \mathrm{~mL}$ of contaminant solution.

This may be attributed to the positively charged surface of Gt at $\mathrm{pH}=6$, which should favor the interaction with negatively charged dyes eosin y, rose bengal, and fluorescein. Similar results are observed for phosphate adsorption [24]. In this case, the maximum retention percentage is around $60 \%$ for the SSO-Gt films. The opposite is observed for methylene blue (cationic dye) that is retained more efficiently in SSO-MMT films.

Owing to these results, it was decided to carry out kinetic studies with two differently charged dyes: eosin y (cationic) and methylene blue (anionic). As mentioned in Section 2, SSO films of the same weight $(0.10 \mathrm{~g})$ were immersed in the dye solutions $(3.5 \mathrm{~mL})$ and the absorption of the dyes was automatically recorded as a function of time. Results, represented as the decrease of the (normalized) concentration of the dyes versus time, are shown in Figures 8 and 9, for eosin $\mathrm{y}$ and methylene blue, respectively.

After 4 hours, only a small adsorption of eosin y is observed for SSO and SSO-MMT films. In contrast, an adsorption of $\sim 80 \%$ is observed for SSO-Gt (Figure 8 ). The inset in Figure 8 shows the decrease of eosin y in solution with time due to its adsorption on the SSO-Gt films.

In the case of methylene blue (Figure 9), only SSO-MMT film is shown to be an effective sorbent for the dye. However the amount of compound adsorbed is low; this could be attributed to the solid matrix that limits the expansion of

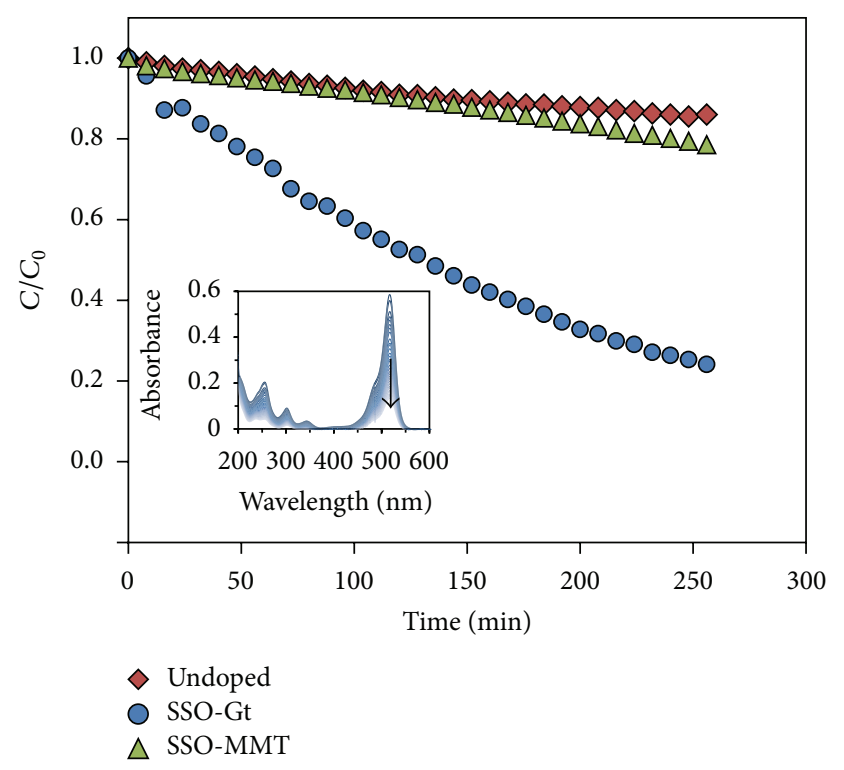

FIGURE 8: Adsorption kinetics of eosin y $(4.0 \mathrm{mg} / \mathrm{L})$ on undoped SSO film (red rhombus), SSO-Gt film (blue circle), and SSO-MMT film (green triangle). Inset: change in UV-Vis absorption spectra of eosin $y$ in the presence of SSO-Gt film as a function of time.

the clay and reduces its ability as sorbent of cationic compounds. 


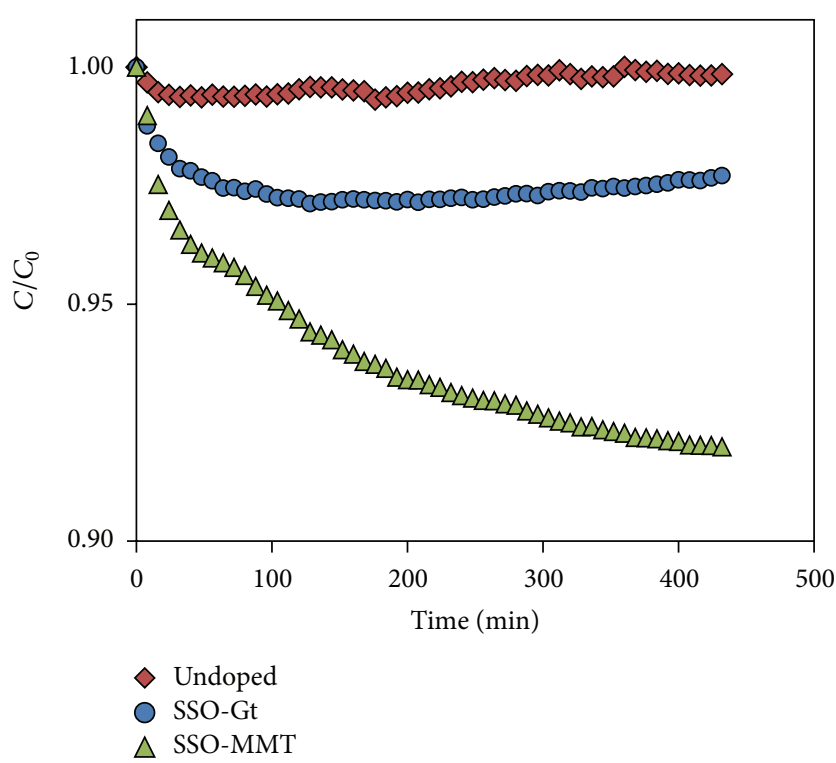

FIGURE 9: Adsorption kinetics of methylene blue $(4.2 \mathrm{mg} / \mathrm{L})$ on films: SSO (red rhombus), SSO-Gt (blue circle), and SSO-MMT (green triangle).

\section{Conclusions}

SSO with pendant dodecyl chains undoped and doped with Gt or MMT were synthesized and characterized. Experimental results showed that it is possible to obtain nearly homogeneous dispersion of the particles in the matrix. Characterization tests confirmed this, noting the distinctive bands of Gt and MMT onto SSO films by SEM-EDAX, XRD, FTIR, and DSC analysis.

Adsorption experiments showed that the synthesized materials are able to retain metal cations, organic dyes, and phosphate, making these films useful for removing different types of environmental contaminants. In particular, undoped films or films doped with Gt would be suitable for the retention of anionic species, while SSO-MMT films could be used for trapping preferentially cations. Finally, it is important to note that, in contrast to the use of adsorbent suspensions for the decontamination of wastewater (which always requires further processing to separate the sorbent particles of the medium being treated, such as induced precipitation, decantation, and centrifugation), the SSO films synthesized in this study have the large advantage that they can be easily recuperated after treatment. In other words, these films could be applied as simple surfaces contaminant trappers, which would facilitate the adsorption of contaminants.

\section{Conflict of Interests}

The authors declare that there is no conflict of interests regarding the publication of this paper.

\section{Acknowledgments}

The authors thank Cristina Zenobi (Universidad Nacional del Sur) for her help with the atomic absorption measurements.
Universidad Nacional de Río Cuarto (PPI 18/C390 and PPI 18/C421), Consejo Nacional de Investigación Científica y Tecnológica (PIP 11220100100284), and Agencia Nacional de Promoción Científica y Tecnológica (PICT 2691/2011 and PICT 1439/2013) are gratefully acknowledged for the financial support.

\section{References}

[1] K. J. Shea, D. A. Loy, and O. W. Webster, "Arylsilsesquioxane gels and related materials. New hybrids of organic and inorganic networks," Journal of the American Chemical Society, vol. 114, no. 17, pp. 6700-6710, 1992.

[2] D. A. Loy and K. J. Shea, "Bridged polysilsesquioxanes. Highly porous hybrid organic-inorganic materials," Chemical Reviews, vol. 95, no. 5, pp. 1431-1442, 1995.

[3] K. J. Shea and D. A. Loy, "Bridged polysilsesquioxanes. Molecular-engineered hybrid organic-inorganic materials," Chemistry of Materials, vol. 13, no. 10, pp. 3306-3319, 2001.

[4] L. Nicole, C. Laberty-Robert, L. Rozes, and C. Sanchez, "Hybrid materials science: a promised land for the integrative design of multifunctional materials," Nanoscale, vol. 6, no. 12, pp. 62676292, 2014.

[5] P. Gómez-Romero and C. Sanchez, Functional Hybrid Materials, Wiley-VCH, Weinheim, Germany, 2004.

[6] C. Sanchez, B. Julián, P. Belleville, and M. Popall, "Applications of hybrid organic-inorganic nanocomposites," Journal of Materials Chemistry, vol. 15, no. 35-36, pp. 3559-3592, 2005.

[7] A. Dabrowski and M. Barczak, "Bridged polysilsesquioxanes as a promising class of adsorbents. A concise review," Croatica Chemica Acta, vol. 80, no. 3-4, pp. 367-380, 2007.

[8] M. C. Burleigh, M. A. Markowitz, M. S. Spector, and B. P. Gaber, "Amine-functionalized periodic mesoporous organosilicas," Chemistry of Materials, vol. 13, no. 12, pp. 4760-4766, 2001.

[9] A. I. Kirillov, E. V. Panezhda, L. I. Belousova et al., "Uranium(VI) sorption on polysilsesquioxanes with various organic functional groups," Russian Journal of Applied Chemistry, vol. 75, no. 5, pp. 724-726, 2002.

[10] Y. N. Pozhidaev, E. V. Panezhda, O. Y. Grigor'eva et al., "Organosilicon sorbents for lanthanides," Doklady Chemistry, vol. 393, no. 4-6, pp. 283-286, 2003.

[11] A. Dabrowski, M. Barczak, E. Robens et al., "Ethylene and phenylene bridged polysilsesquioxanes functionalized by amine and thiol groups as adsorbents of volatile organic compounds," Applied Surface Science, vol. 253, no. 13, pp. 5747-5751, 2007.

[12] M. L. Gómez, D. P. Fasce, R. J. J. Williams, H. A. Montejano, and C. M. Previtali, "Photoluminescence of bridged polysilsesquioxanes containing urea groups: doping with safranine-o to generate red-light emission," Journal of Polymer Science Part B: Polymer Physics, vol. 46, no. 3, pp. 289-296, 2008.

[13] M. L. Gómez, C. E. Hoppe, H. A. Zucchi, R. J. J. Williams, M. I. Giannotti, and M. A. López-Quintela, "Hierarchical assemblies of gold nanoparticles at the surface of a film formed by a bridged silsesquioxane containing pendant dodecyl chains," Langmuir, vol. 25, no. 2, pp. 1210-1217, 2009.

[14] R. J. J. Williams, C. E. Hoppe, I. A. Zucchi et al., "Self-assembly of nanoparticles employing polymerization-induced phase separation," Journal of Colloid and Interface Science, vol. 431, pp. 223-232, 2014.

[15] M. L. Gómez, I. E. dell'Erba, C. A. Chesta, C. E. Hoppe, and R. J. J. Williams, "Dispersion of gold dodecanethiolate 
in a silsesquioxane film with pendant dodecyl chains: from photoluminescent materials to gold nanocomposites," Journal of Materials Science, vol. 48, no. 24, pp. 8559-8565, 2013.

[16] M. L. Gómez, C. E. Hoppe, and R. J. J. Williams, "In situ generation of silver microstructures by thermal decomposition of silver $n$-dodecanethiolate dispersed in an organic-inorganic hybrid coating," Materials Chemistry and Physics, vol. 130, no. 1-2, pp. 519-523, 2011.

[17] M. G. Alvarez, M. L. Gómez, S. J. Mora, M. E. Milanesio, and E. N. Durantini, "Photodynamic inactivation of Candida albicans using bridged polysilsesquioxane films doped with porphyrin," Bioorganic \& Medicinal Chemistry, vol. 20, no. 13, pp. 40324039, 2012.

[18] R. M. Spada, M. Cepeda-Plaza, M. L. Gómez et al., "Clean singlet oxygen production by a $\operatorname{Re}(\mathrm{I})$ complex embedded in a flexible self-standing polymeric silsesquioxane film," The Journal of Physical Chemistry C, vol. 119, no. 18, pp. 10148-10159, 2015.

[19] W. Stumm, Chemistry of the Solid-Water Interface: Processes at the Mineral-Water and Particle-Water Interface in Natural Systems, John Wiley \& Sons, 1992.

[20] R. Srinivasan, "Advances in application of natural clay and its composites in removal of biological, organic, and inorganic contaminants from drinking water," Advances in Materials Science and Engineering, vol. 2011, Article ID 872531, 17 pages, 2011.

[21] C. V. Waiman, M. J. Avena, A. E. Regazzoni, and G. P. Zanini, "A real time in situ ATR-FTIR spectroscopic study of glyphosate desorption from goethite as induced by phosphate adsorption: effect of surface coverage," Journal of Colloid and Interface Science, vol. 394, no. 1, pp. 485-489, 2013.

[22] G. P. Zanini, C. Maneiro, C. Waiman, J. A. Galantini, and R. A. Rosell, "Adsorption of metsulfuron-methyl on soils under notill system in semiarid Pampean Region, Argentina," Geoderma, vol. 149, no. 1-2, pp. 110-115, 2009.

[23] C. V. Waiman, M. J. Avena, M. Garrido, B. F. Band, and G. P. Zanini, "A simple and rapid spectrophotometric method to quantify the herbicide glyphosate in aqueous media. Application to adsorption isotherms on soils and goethite," Geoderma, vol. 170, pp. 154-158, 2012.

[24] D. L. Sparks, Environmental Soil Chemistry, Academic Press, New York, NY, USA, 2nd edition, 2003.

[25] R. J. Atkinson, A. M. Posner, and J. P. Quirk, "Adsorption of potential-determining ions at the ferric oxide-aqueous electrolyte interface," Journal of Physical Chemistry, vol. 71, no. 3, pp. 550-558, 1967.

[26] M. Kosmulski, S. Durand-Vidal, E. Maczka, and J. B. Rosenholm, "Morphology of synthetic goethite particles," Journal of Colloid and Interface Science, vol. 271, no. 2, pp. 261-269, 2004.

[27] R. Ilari, M. Etcheverry, C. Zenobi, and G. Zanini, "Effect of the surfactant benzalkonium chloride in the sorption of paraquat and cadmium on montmorillonite," International Journal of Environment and Health, vol. 7, no. 1, pp. 70-82, 2014.

[28] E. M. Pecini and M. J. Avena, "Measuring the isoelectric point of the edges of clay mineral particles: the case of montmorillonite," Langmuir, vol. 29, no. 48, pp. 14926-14934, 2013.

[29] J. Murphy and J. P. Riley, "A modified single solution method for the determination of phosphate in natural waters," Analytica Chimica Acta, vol. 27, pp. 31-36, 1962.
[30] A. F. Gualtieri and P. Venturelli, "In situ study of the goethitehematite phase transformation by real time synchrotron powder diffraction," American Mineralogist, vol. 84, no. 5-6, pp. 895904, 1999.

[31] C. Luengo, V. Puccia, and M. Avena, "Arsenate adsorption and desorption kinetics on a Fe(III)-modified montmorillonite," Journal of Hazardous Materials, vol. 186, no. 2-3, pp. 1713-1719, 2011.

[32] T. Grewer, "The influence of chemical structure on exothermic decomposition," Thermochimica Acta, vol. 187, pp. 133-149, 1991.

[33] U. Schwertmann and R. M. Cornell, Iron Oxides in the Laboratory: Preparation and Characterization, Wiley-VCH, Weinheim, Germany, 2nd edition, 2000.

[34] B. J. Lemaire, P. Davidson, J. Ferre et al., "The complex phase behaviour of suspensions of goethite $(\alpha-\mathrm{FeOOH})$ nanorods in a magnetic field," Faraday Discussions, vol. 128, pp. 271-283, 2005.

[35] Y. Lv, X. Su, F. Ren, H.-Q. Kan, and Y. Kong, "Analysis of conditions for the preparation of BSA/MMT composites," Science and Engineering of Composite Materials, vol. 20, no. 3, pp. 221-226, 2013.

[36] R. Wang, G. Baran, and S. L. Wunder, "Packing and thermal stability of polyoctadecylsiloxane compared with octadecylsilane monolayers," Langmuir, vol. 16, no. 15, pp. 6298-6305, 2000. 

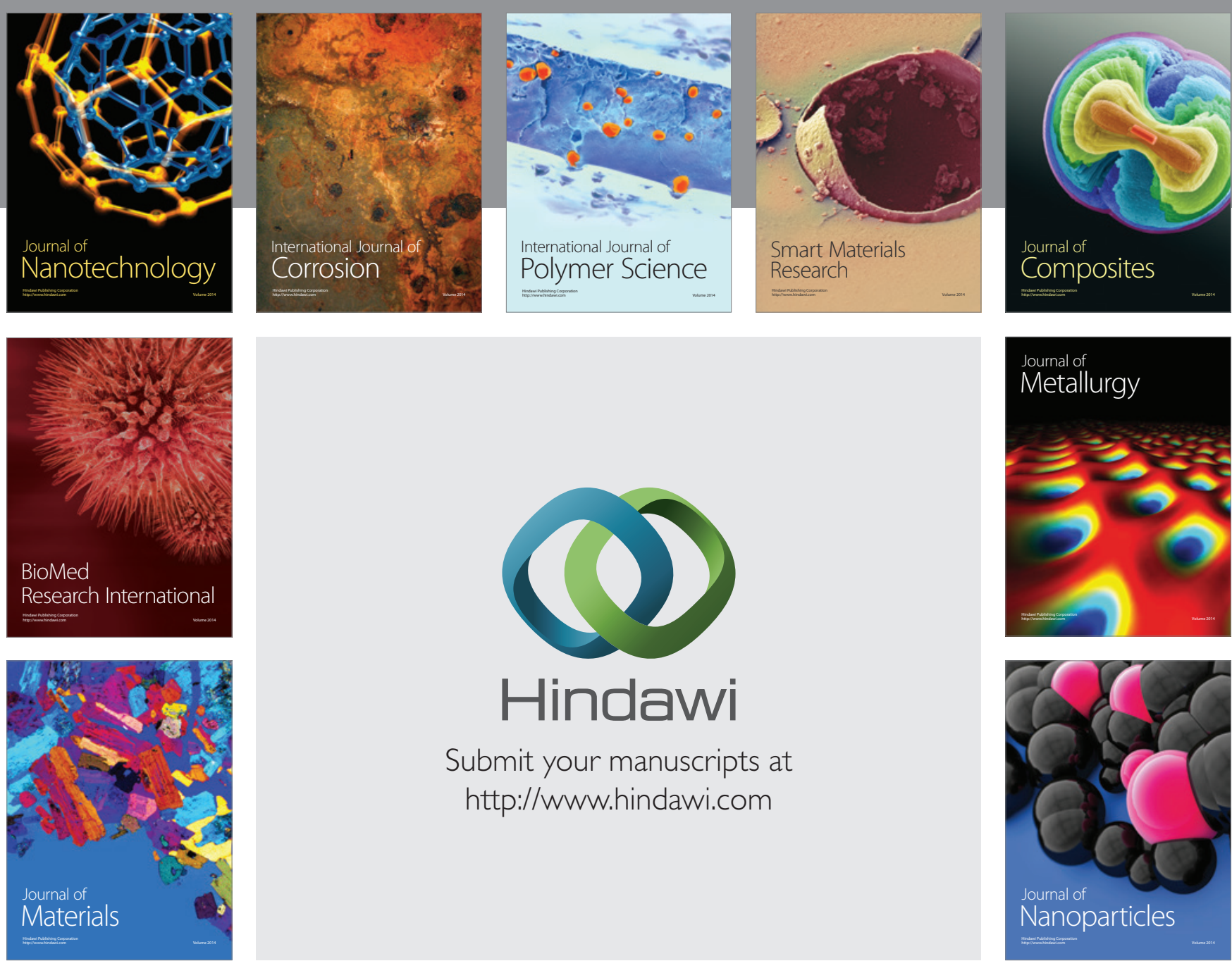

\section{Hindawi}

Submit your manuscripts at

http://www.hindawi.com

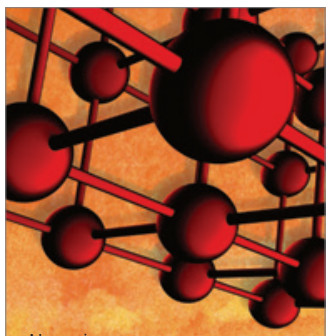

Materials Science and Engineering
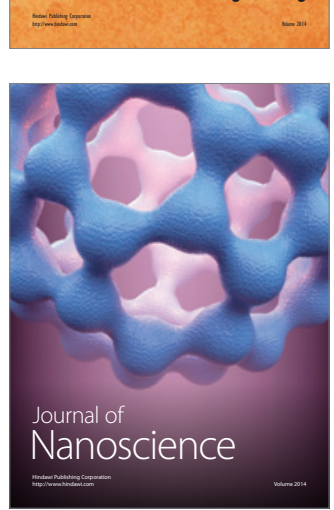
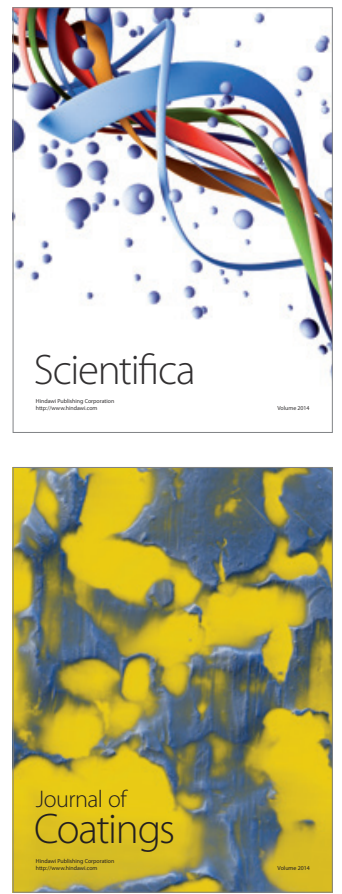
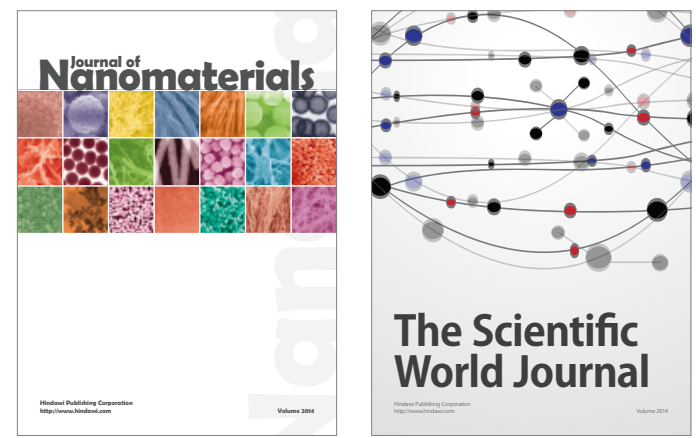

The Scientific World Journal
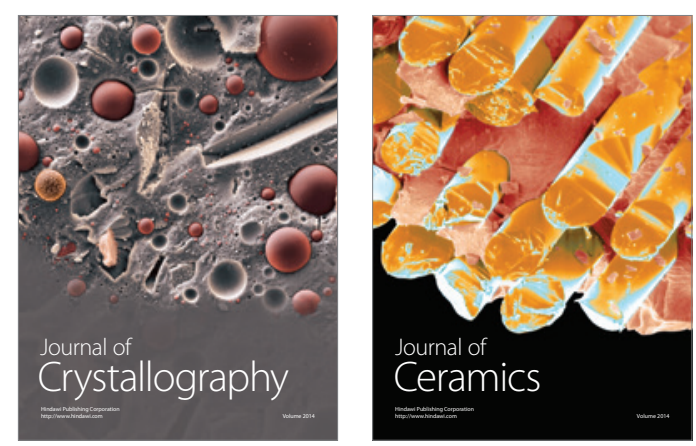
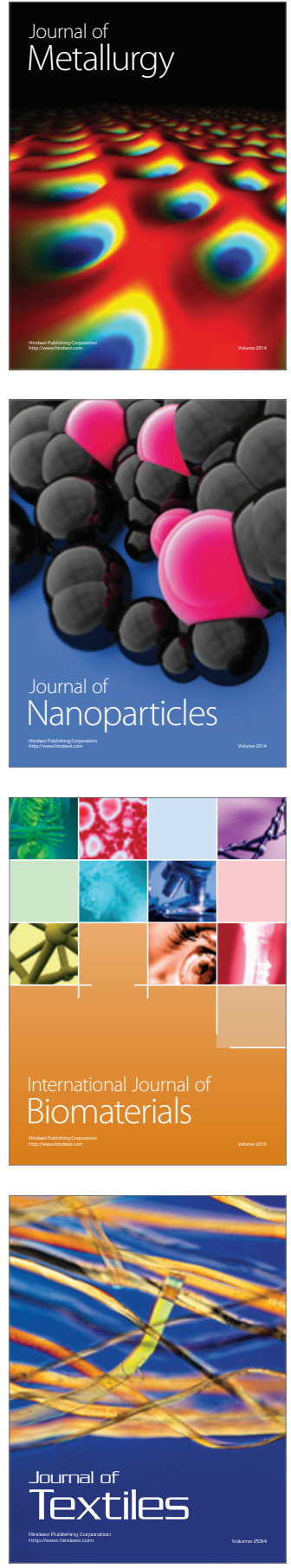